Disponível em:

http://editora.unoesc.edu.br/index.php/race

RACE, Joaçaba, p. 131-156, Edição Especial. 2017

\title{
A CULTURA ORGANIZACIONAL COMO IMPULSIONADORA DOS PROCESSOS DE INTELIGÊNCIA NA GESTÃO PÚBLICA
}

Organizational culture as a driver of intelligence processes in public management

Claudia Melati

E-mail: cmelati@yahoo.com.br

Pós-graduada em Administração pública Contemporânea pela Universidade Federal do Rio Grande do Sul; Mestranda do Programa de Pós-Graduação em Administração da Escola de Administração na Universidade Federal do Rio Grande do Sul; Servidora Pública do Estado do Rio Grande do Sul.

Raquel Janissek-Muniz

E-mail: rjmuniz@ufrgs.br

Doutora em Gestão pela Université Pierre Mendes France de Grenoble, França; Mestre em Administração pela Universidade Federal do Rio Grande do Sul; Professora do Programa de Pós-Graduação em Administração da Escola de Administração na Universidade Federal do Rio Grande do Sul. Endereço para contato: Avenida Paulo Gama, 110, Farroupilha, 90040-060, Porto Alegre, Rio Grande do Sul, Brasil.

Artigo recebido em 30 de julho de 2017. Aceito em 13 de novembro de 2017. 


\section{Resumo}

O uso de dados e informações advindos do ambiente externo à administração pública pode proporcionar o aprimoramento da tomada de decisão dos gestores públicos, bem como uma maior eficiência e eficácia no desenvolvimento da atividade pública. Sistematizar o monitoramento de ambientes exige a estruturação de processos de inteligência, os quais possuem foco nas informações externas das organizações, objetivando o monitoramento do ecossistema como um todo, sendo determinante para as organizações conhecerem e se adaptarem ao seu ambiente. Reconhecendo a importância de uma cultura organizacional impulsionadora que permita a manutenção e a evolução desses processos, neste estudo buscou-se identificar pontos relevantes relacionados à influência da cultura organizacional na concretização de processos de inteligência na gestão pública. Com a realização de 17 entrevistas com gestores públicos de diferentes, foi possível evidenciar que os mesmos reconhecem a cultura organizacional como impulsionadora dos processos de inteligência, uma vez que apontam o fortalecimento de um ambiente estimulado para o monitoramento do ambiente externo, o engajamento das pessoas e a utilização de tecnologias da informação e comunicação como mecanismos-chave da cultura organizacional para a concretização de tais processos. Identifica-se como limitação deste trabalho a não realização de entrevistas com gestores que trabalham diretamente com processos de inteligência, bem como a participação de órgãos de um único estado no estudo, impossibilitando a realização de comparativos entre culturas organizacionais. Para estudos futuros, sugere-se a continuidade da pesquisa por meio da realização de entrevistas com servidores que não exerçam cargos de gestão.

Palavras-chave: Cultura organizacional. Inteligência. Gestão pública.

\section{Abstract}

The use of data and information from the environment outside the Public Administration can provide the improvement of the decision-making of public managers, as well as greater efficiency and effectiveness in the development of public activity. Systematizing the monitoring of environments requires the structuring of Intelligence processes, which have a focus on the external information of the organizations, aiming at the monitoring of the ecosystem as a whole, being determinant for the organizations to know and adapt to their environment. Recognizing the importance of a driving organizational culture that allows the maintenance and evolution of such processes, this study sought to identify relevant points related to the influence of organizational culture in the implementation of Intelligence processes with Public Management. With the accomplishment of 17 interviews with different public managers, it was possible to show that they recognize the organizational culture as the driver of the Intelligence processes, since they point to the strengthening of an environment stimulated for the monitoring of the external environment, the engagement of people and the use of information and communication technologies as key mechanisms of organizational culture for the accomplishment of such 
processes. It is identified as a limitation of this work not to conduct interviews with managers who work directly with Intelligence processes, as well as, the participation of Agency of a single State in the study, not making it possible to perform comparative among organizational cultures. For future studies, it is suggested to continue the research by conducting interviews with servers that do not hold management positions.

Keywords: Organizational culture. Intelligence. Public management.

\section{INTRODUÇÃO}

A gestão pública no Brasil está em processo de busca da redefinição do papel do Estado por meio da Reforma Gerencial do Estado Brasileiro. Tal processo ocorre desde meados dos anos 1990 e se baseia na descentralização do aparelho de Estado, buscando separar as atividades de planejamento e de execução do Governo por meio da privatização das estatais, da terceirização dos serviços públicos e da criação de organismos de regulação das atividades públicas conduzidas pelo setor público. Além disso, o setor público procura adotar algumas ferramentas gerenciais e ideias provenientes do setor privado, como por exemplo, a reengenharia, o downsizing, as melhores práticas, a gestão do conhecimento, os processos de inteligência, entre outros, como mecanismos para melhorar a prestação do serviço público (BATISTA, 2012; DE PAULA, 2005; RIBEIRO; PEREIRA; BENEDICTO, 2013). A orientação está na construção de uma administração pública mais participativa, eficiente e integrada em rede (CAPOBIANGO, 2013; DE PAULA, 2005; SECCHI, 2009), propiciando, assim, uma gestão de qualidade para a entrega de serviços à sociedade.

Para Ribeiro, Pereira e Benedicto (2013), as reformas da administração pública contribuíram para, além da descentralização do Estado, melhorar a capacidade de decisão política, com a implementação da coordenação horizontal e da modernização do potencial humano de gestão. Alguns fatores importantes para a modernização da administração pública estão nas diversas ações relacionadas ao programa de Governo Eletrônico (eGOV), por meio do uso de tecnologias da informação e comunicação (TICs) e da melhoria da eficiência dos processos operacionais e administrativos dos governos (AGUNE; CARLOS, 2005; OSBORNE; PLASTRIK, 1997). A partir do uso intensivo de TICs na gestão pública, houve um aumento considerável de dados e informações demandados pela sociedade, bem como aqueles produzidos nas organizações públicas. Esse contexto requisitou da gestão pública uma busca pela melhoria da gestão de dados e informações advindos do ambiente, como forma de aprimorar 
a tomada de decisão do gestor e aumentar a eficiência e eficácia da gestão pública (MELATI; JANISSEK-MUNIZ, 2017).

Segundo pesquisa da Forrester Research, publicada na revista InformationWeek Government em 2014, as agências governamentais devem encontrar melhores maneiras de aproveitar dados para atender às expectativas dos cidadãos e para prestar serviços em uma era de volume de dados sem precedentes e conectividade móvel generalizada. Ainda, segundo a pesquisa, nesse contexto governos inteligentes tornam-se fortes jogadores, mostrando que eles podem melhorar o acesso a informações e serviços, facilitar a mobilidade por meio de novos canais e transformar dados em insights e inovação para atender às necessidades dos cidadãos. A pesquisa explicita a importância no desenvolvimento de processos de inteligência na gestão pública como mecanismo impulsionador para a realização da atividade pública e de seus objetivos finalísticos junto à sociedade.

A partir do contexto apresentado, reforça-se a questão da informação poder ser considerada o bem mais valioso das organizações (FREITAS; JANISSEK-MUNIZ, 2006), além de ser considerada a matéria-prima do trabalho gerencial (AUSTER; CHOO, 1994; RODRIGUES; BLATTMANN, 2014). A obtenção de dados ou de informações torna-se um pressuposto básico, e o diferencial depende de uma nova postura de gestão diante dos insumos informacionais potencialmente disponíveis, advindos do ambiente pertinente da organização, e que se atualizam a todo instante (JANISSEK-MUNIZ; BLANCK, 2014).

Diversos estudos sobre a importância da informação como impulsionadora da tomada de decisão gerencial (DAVENPORT, 1998; CHOO, 2002; ANDRIOTTI; FREITAS; JANISSEK-MUNIZ; 2008; DE PAULA; ROVER, 2012) apontam que há a necessidade de um monitoramento do ambiente externo ao qual a organização pertence. Esse processo é referenciado como a atividade de inteligência das organizações e, por envolver percepção, obtenção, seleção e análise de dados interpretados e contextualizados, requer uma intervenção e ação humana (DAVENPORT, 1998).

Para que o processo de inteligência tenha êxito na gestão pública, questiona-se a respeito da adaptação da cultura organizacional nos órgãos públicos, uma vez que, por muitos anos, a modernização administrativa esteve estanque, e o olhar estava direcionado para dentro da organização. A preocupação da gestão pública estava centrada no desenvolvimento das atividades burocráticas em detrimento de uma visão mais ampla de monitoramento de dados e informações úteis e que pudessem auxiliar na construção de um Estado mais eficiente em seu dever para com a socie- 
dade (DESOUZA, 2005; JOHNSTON; HANSEN, 2011; GIL-GARCIA; ZHANG; PURON-CID, 2016; BATISTA, 2012; KLERING; PORSSE; GUADAGNIN, 2010).

Segundo Bergue (2010), a gestão organizacional nas organizações públicas brasileiras possui um componente definido por aspectos de natureza histórica, identificados pelos valores da organização, por elementos culturais de forma geral, além de aspectos constitucionais e legais. Para o autor, alguns aspectos merecem destaque como, por exemplo, a menor suscetibilidade do ambiente a turbulências, constância no ritmo e dinâmica de trabalho, segurança na manutenção do vínculo laboral, valorização dos padrões formais, corporativismo e condescendência. Estes são componentes culturais que precisam ser considerados por ocasião da proposição de mudanças nas organizações, ainda que o arranjo cultural específico de cada uma seja único.

Dessa forma, quando nos deparamos com a estruturação de novos processos de gestão, como os de inteligência na administração pública, precisamos compreender de que forma questões relacionadas à cultura organizacional influenciam e são determinantes para o sucesso desses processos. De fato, é sabido que a implantação e a manutenção de processos de inteligência, definidos como a capacidade que a organização tem de monitorar seu ambiente externo e considerar elementos informacionais na sua tomada de decisão, demandam envolvimento adequado por parte da organização, a qual deve possuir a capacidade antecedente de se preparar para possíveis fatores críticos que possam determinar o sucesso ou o fracasso da atividade (JANISSEK-MUNIZ, 2016). Segundo a autora, entre os diversos fatores críticos que os projetos de inteligência possuem em comum, pode-se citar a gestão de recursos humanos dentro do projeto; o envolvimento da alta direção, a qual deve sustentar, apoiar, legitimar e manifestar interesse no projeto durante toda sua duração sem anular as partes envolvidas; a importância de uma mobilização constante das partes envolvidas no projeto; o comportamento dos membros na condução do projeto; e o turnover da equipe de projeto, que pode impactar na continuidade do projeto. Enfim, esses são alguns dos fatores determinantes em termos de tornar perene e contínuo um dispositivo de inteligência.

Diante desse contexto, no presente estudo visa-se identificar pontos importantes relacionados à influência da cultura organizacional na concretização de processos de inteligência na gestão pública. Para isso, buscou-se realizar uma minuciosa revisão de literatura sobre os conceitos norteadores deste estudo. Posteriormente, realizaram-se entrevistas semiestruturadas com 17 gestores públicos de diversas áreas de atuação, considerando que o trabalho de captação e utilização de dados e informações advindos do ambiente possui maior interligação com áreas estratégicas e pessoas-chave para a tomada de decisão. 
Além dessa introdução, o artigo apresenta uma revisão da literatura sobre o conceito de inteligência e a forma como ela está sendo estudada na gestão pública, enfatizando-se aspectos relacionados à cultura organizacional. Em seguida, são apresentados os procedimentos metodológicos, culminando com a apresentação e discussão dos resultados obtidos. Por fim, são apresentadas as considerações finais, com as limitações do estudo e as recomendações para estudos futuros.

\section{REFERENCIAL TEÓRICO}

O entendimento da importância da cultura organizacional como suporte ao estabelecimento de processos de inteligência em organizações públicas é um terreno ainda pouco explorado, conforme evidenciou-se com o levantamento teórico realizado no presente trabalho. Pensando nisso, o arcabouço teórico que suporta esta pesquisa foi estruturado sobre os conceitos relacionados ao monitoramento do ambiente e a utilização de dados e informações pela gestão pública, inerentes ao processo de inteligência, bem como em relação à cultura organizacional. Nas seções a seguir, abordam-se esses conceitos, visando estabelecer a relação pretendida no desenvolvimento deste artigo.

\subsection{CULTURA ORGANIZACIONAL}

A cultura organizacional pode ser entendida como um padrão de suposições básicas compartilhadas, que foi aprendido por um grupo à medida que ele solucionava seus problemas de adaptação externa e integração interna, ao passo do referido padrão funcionar bem o suficiente para ser considerado válido e ser ensinado aos novos membros como o modo correto de perceber, pensar e sentir em relação a esses problemas (SCHEIN, 2010). Russo (2010) explica que esse padrão de suposições básicas diz respeito aos valores que regem o comportamento, os quais vão sendo internalizados e se tornando inconscientes a ponto de passarem a ser considerados naturais.

Segundo Hofstede (1991), a cultura organizacional relaciona-se com a história e a tradição da organização, sendo de difícil decifração e explicação; é, por natureza, coletiva e relaciona-se a valores, crenças, conhecimentos e outros aspectos mais profundos. Para o autor, a cultura cria distinção entre uma organização e as demais, transmite um senso de identidade para seus membros, facilita o comprometimento com algo maior que o interesse individual e aumenta a estabilidade do sistema social, 
uma vez que fornece padrões sobre o que os funcionários devem ou não fazer, moldando as atitudes e comportamentos dos empregados.

De acordo com Robbins e Judge (2012), uma cultura organizacional forte, compreendida como aquela em que a maioria dos empregados possui as mesmas opiniões sobre a missão e os valores da organização, proporciona estabilidade a uma organização, podendo ser, ao mesmo tempo, uma barreira à mudança, uma vez que ela tem grande influência sobre as atitudes e os comportamentos de seus membros. Segundo os autores, o grau com que os funcionários são estimulados a inovar e assumir riscos, o grau que se espera que os funcionários demonstrem atenção aos detalhes, o foco nos resultados, a consideração em torno dos efeitos dos resultados sobre as pessoas na organização, a orientação para a equipe, o grau de competição e agressividade das pessoas e, por fim, o quanto a organização enfatiza a manutenção do status quo diante do crescimento são as sete características básicas que capturam a essência da cultura de uma organização.

Em termos práticos, a cultura organizacional descreve o ambiente em que as pessoas trabalham, bem como a influência deste sobre a forma como elas pensam, atuam e experimentam o trabalho (WARRICK; MILLIMAN; FERGUSON, 2016). Ainda, a "cultura organizacional se refere a um sistema de valores compartilhados pelos membros que diferenciam uma organização das demais.” (ROBBINS, 2005, p. 375).

Quando pensamos no contexto de organizações públicas brasileiras, algumas características básicas podem ser atribuídas, como a incorporação de traços de paternalismos, alguns deles entranhados na própria legislação, como licenças, vantagens e concessões que beneficiam excessivamente alguns agentes em relação à média; é também marcadamente heterogênea, se compararmos os diferentes espaços organizacionais dentro do serviço público (BERGUE, 2010).

Embora a cultura organizacional da administração pública possa ter as referidas características básicas, é inerente a existência de incontáveis subculturas dentro dos mais diversos órgãos públicos. Segundo Robbins (2005, p. 377), a maioria das organizações possui uma cultura dominante, que expressa os valores essenciais compartilhados pela maioria dos mesmos; além disso, possui diversas subculturas que refletem problemas, situações ou experiências de alguns membros ou setores da organização, e estas subculturas não podem ser desconsideradas nem descartadas.

Segundo Ferreira et al. (2002), a análise de alguns dos principais instrumentos desenvolvidos para a avaliação das manifestações da cultura organizacional revela que eles enfatizam, prioritariamente, os traços culturais associados aos valores, às normas comportamentais e às práticas organizacionais, e vêm sendo adotados fre- 
quentemente nos estudos destinados a elucidar o impacto que a cultura das organizações exerce nas atitudes e nos comportamentos expressos pelos indivíduos que as integram.

Com os anos, a cultura organizacional passou por transformações inerentes à evolução de questões sociais e tecnológicas, entre outros fatores inerentes aos processos organizacionais. Contudo, segundo Bergue (2011), o processo de mudança nos padrões culturais da organização não admite rupturas bruscas, mas transformações adaptativas operadas em vetores essenciais da estrutura. A cultura de uma organização é tomada como uma estrutura de valores bastante complexa, que não somente pode, mas deve ser gerenciada, de tal forma a orientá-la para os objetivos institucionais (BERGUE, 2011; SCHEIN, 2010; ROBBINS, 2005).

O aumento considerável de produção de dados e informações, a intensificação do uso de tecnologias da informação e comunicação, a maior fiscalização social e exigência de uma melhor prestação do serviço público, entre outros fatores, impulsionaram a administração pública a revisar sua cultura rígida no sentido de adaptabilidade às necessidades e mudanças trazidas pela sociedade por meio das novas gerações. Entre os novos conceitos e processos, pode-se destacar a atividade de inteligência na gestão pública.

\subsection{INTELIGÊNCIA NA GESTÃO PÚBLICA}

Dados e informações são gerados a todo instante por pessoas, instituições públicas e empresas no ambiente externo à organização (PRESCOTT; MILLER, 2002). Por meio do monitoramento que surgem informações que podem potencialmente sinalizar mudanças de toda ordem, como, por exemplo, alterações no ambiente de negócios e no ambiente tecnológico, entre outras (DAVENPORT, 1998; JANISSEK-MUNIZ; BLANCK, 2014). A diversidade de elementos ambientais que precisam ser monitorados requer um esforço significativo de gestores e tomadores de decisão (VIDIGAL; ZIVIANI, 2015). De fato, a compreensão de sua existência e a obtenção do conhecimento deles decorrente é importante para a tomada de decisão dos gestores, independentemente do contexto de atuação.

O monitoramento do ambiente consiste na busca por informações no ambiente organizacional, objetivando apoiar a organização no planejamento e na tomada de decisão estratégicos (ANDRIOTTI; FREITAS; JANISSEK-MUNIZ, 2008). Aguilar (1967), já na década de 1960, definiu o monitoramento estratégico como a atividade de adquirir, no ambiente externo das organizações, o conhecimento necessário aos tomadores de decisão para traçar o futuro curso da ação. Segundo Choo (1999), o 
monitoramento deve ser realizado como um processo planejado, sustentável e coordenado. A sistematização do monitoramento de ambiente ocorre por meio de processos de inteligência, uma vez que "é exatamente a partir dos dados, do significado da informação que resulta destes e do conhecimento advindo a partir da compreensão, entendimento e aprendizado proporcionados pelo conhecimento que a Inteligência pode se estabelecer.” (JANISSEK-MUNIZ; BLANCK, 2014, p. 8).

Conforme Lesca (2003), inteligência é a capacidade que uma organização possui de, a partir do monitoramento proativo do seu ambiente pertinente externo, fazer uso da informação, obtida e interpretada, para se adaptar às oportunidades ou ameaças detectadas a partir desse monitoramento. O processo de inteligência, que envolve percepção, obtenção, seleção e análise de dados interpretados e contextualizados, é alimentado por informações diversas, obtidas em um ambiente organizacional impulsionado pela era da comunicação, com intensidade crescente de informação à disposição de todos. Como processo, a inteligência deve ser sistemática, formalizada e estruturada, para transformar informações dispersas em inteligência acionável e para criar insights valiosos, os quais serão usados em ações e decisões operacionais e estratégicas (HAKANSSON; NELKE, 2015).

Alguns trabalhos acadêmicos realizados sobre a temática inteligência voltada para a administração pública (CEPIK, 2005, 1997; DESOUZA, 2005; GIL-GARCIA; PARDO; ALDAMA-NALDA, 2013; JIMÉNEZ; SOLANAS; FALCONE, 2014; JOHNSTON; HANSEN, 2011; LINDERS; LIAO; WANG, 2015; BRÂNZAS; RADU, 2015) argumentam que existem algumas questões peculiares do contexto público em relação ao setor privado quando pensamos na atividade de inteligência e ressaltam a importância da realização contínua de monitoramento de ambiente e análise de dados em governo.

Segundo Herman (1996), governos recolhem, processam e utilizam informações, mas o conceito inteligência no governo normalmente tem um significado associado a relações internacionais, defesa, segurança nacional e sigilo, possuindo, inclusive, instituições especializadas rotuladas de inteligência. Corroborando o exposto pelo autor, um número relevante de estudos sobre inteligência e governo possui como foco central a área de segurança nacional (CEPIK, 2005; DE PAULA; ROVER, 2012; DESOUZA, 2005; VERONESE, 2013), principalmente com o objetivo de analisar o papel das agências de inteligências de cada país.

Entretanto, baseando-se em estudos de Cepik (1997), Herman (1996) e Desouza (2005), depreende-se que, de uma vinculação seminal do processo de inteligência às questões restritas de segurança pública e relações internacionais, estudos mais 
recentes mostram uma nova orientação e evolução do processo, atualmente voltado a uma maior abrangência do conceito de inteligência em governo. Segundo certos autores (GIL-GARCIA; PARDO; ALDAMA-NALDA, 2013; GIL-GARCIA; HELBIGI; OJO, 2014; JOHNSTON; HANSEN, 2011; SCHOLL; SCHOLL, 2014)societies and their governments around the world have been meeting unprecedented challenges, many of which surpass the capacities, capabilities, and reaches of their traditional institutions and their classical processes of governing. Among these challenges are the need for an accelerated transition of the global economy from its current fossil fuel basis to renewable energies, the so-called post-carbon era also known as the third industrial revolution, the containment and reduction of government spending and debt financing, the increasing rapidity of market changes, and the expanding lag of timely interventions via traditional lawmaking and government action. While upholding the proven principles of Western democracy, democratic self-governance in 21st century market economies apparently needs to develop new institutional formats and novel mechanisms for staying abreast with the systemic dynamics of a tightly interconnected global society. We claim that actionable and omnipresent information along with its underlying technologies are substantial prerequisites and backbones for developing models of smart (democratic, ao tratar de inteligência no governo, surgem questões relacionadas à eficiência da atividade pública, bem como a entrega de serviços públicos de qualidade para a sociedade, e a participação desta como agente de mudança social, a partir das informações advindas do ambiente.

Uma das principais questões que influenciou fortemente a evolução do entendimento e aplicação de inteligência no governo está no uso de TICs (DE PAULA; ROVER, 2012; GIL-GARCIA; ZHANG; PURON-CID, 2016; JOHNSTON; HANSEN, 2011; SCHOLL; SCHOLL, 2014). Uma das finalidades do uso de TICs no governo está na coleta de dados que podem ser usados para impulsionar melhorias na tomada de decisão e, consequentemente, em uma melhor prestação do serviço público (GIL-GARCIA; PARDO; ALDAMA-NALDA, 2013).

A informação acionável e onipresente, com suas tecnologias subjacentes, é apontada como pré-requisito substancial para o desenvolvimento de modelos de governo inteligente, no qual as instituições governamentais inteligentes são abertas e ágeis, bem como adotam a participação dos interessados em colaborar em todos os níveis e em todos os ramos de governo (SCHOLL; SCHOLL, 2014). Outro ponto passível de caracterizar um governo inteligente está na entrega de serviços ao cidadão, além da eficiência e eficácia dos serviços prestados e na resolução de questões de governo e sociedade, mediante colaboração interdepartamental (LIU; ZHENG, 2015). 
Para Rezende (2012), nas organizações públicas a inteligência pública está relacionada com a utilização de informações e conhecimentos sistematizados, personalizados e oportunos para a tomada de decisão, além disso, uma organização inteligente pode gerar novos serviços, produtos, negócios ou atividades, além dos triviais e, como consequência, contribuir para o seu êxito ou sucesso.

Segundo Johnston e Hansen (2011), a superação de desafios complexos por parte do governo está no aumento da utilização das capacidades coletivas dos indivíduos para organizar, interagir e governar. Assim, os autores sugerem uma infraestrutura de governo inteligente, que é mais ágil e mais eficiente que os paradigmas do governo atual. Ainda, outros estudos argumentam que os fatores-chave para a criação de um governo inteligente, capaz de lidar com a complexidade e incerteza, são a coordenação, o envolvimento continuado, o acesso aos dados abertos e às informações compartilhadas (GIL-GARCIA; HELBIG; OJO, 2014; SCHOLL; SCHOLL, 2014).

Para Scholl e Scholl (2014), os elementos que caracterizam um governo inteligente estão vinculados à abertura e tomada de decisão; a partilha de informação aberta e o uso das informações; a participação das partes interessadas e colaboração, objetivando a melhoria das operações e serviços governamentais na busca por inovação, sustentabilidade, competitividade e habitabilidade. Para Johnston e Hansen (2011), o ponto-chave de um governo inteligente está em aproveitar, efetivamente, as habilidades e conhecimentos que muitas vezes estão latentes na multidão. Ainda, as TICs são ferramentas que auxiliam no processo de gestão, organização, tratamento e disseminação da informação e construção do conhecimento para as organizações (DE PAULA; ROVER, 2012), potencializando uma melhor tomada de decisão e uma melhor prestação de serviços por parte do governo (GIL-GARCIA; PARDO; ALDAMA-NALDA, 2013).

Ressalta-se que a tomada de decisão baseada em evidências é uma dimensão essencial para caracterizar inteligência em governo, uma vez que decisões baseadas em dados e o uso intensivo deles, por meio do sensoriamento onipresente, medição avançada e aplicação integrada, permite aos governos uma tomada de decisão mais informada e acaba por melhorar a eficácia das políticas e programas públicos (DESOUZA, 2005; GIL-GARCIA; ZHANG; PURON-CID, 2016; SCHOLL; SCHOLL, 2014).

Diante do exposto, evidenciam-se alguns pontos que merecem destaque quando buscamos compreender como se estabelecem os processos de inteligência na gestão pública: o apoio da alta gestão para a concretização de processos de inteligência, o papel das pessoas nos referidos processos e a influência da tecnologia na concretização deles, todos interligados objetivando uma melhor tomada de decisão dos 
gestores públicos e uma maior eficiência e eficácia no desenvolvimento da atividade pública, conforme Quadro 1.

Quadro 1 - Dimensões para o processo de inteligência na gestão pública

\begin{tabular}{|c|c|c|}
\hline Perspectiva & Autores & Pressupostos teóricos \\
\hline $\begin{array}{l}\text { Apoio da } \\
\text { alta gestão }\end{array}$ & $\begin{array}{l}\text { Gil-Garcia, Pardo e Aldama- } \\
\text {-Alda (2013); Gil-Garcia, } \\
\text { Zhang e Puron-Cid (2016); } \\
\text { Johnston e Hansen (2011); } \\
\text { Scholl e Scholl (2014) }\end{array}$ & $\begin{array}{l}\text { Incentivo por parte dos gestores no sentido de criar } \\
\text { uma cultura organizacional voltada para o monito- } \\
\text { ramento do ambiente com o objetivo de captação de } \\
\text { dados e informações pertinentes na construção de uma } \\
\text { administração pública mais eficiente. }\end{array}$ \\
\hline $\begin{array}{l}\text { O capital } \\
\text { humano } \\
\text { (pessoas) }\end{array}$ & $\begin{array}{l}\text { De Paula e Rover (2012); } \\
\text { Gil-Garcia, Pardo e Aldama- } \\
\text {-Alda (2013); } \\
\text { Gil-Garcia, Zhang e Puron- } \\
\text {-Cid (2016); Johnston e Han- } \\
\text { sen (2011); Scholl e Scholl } \\
\text { (2014) }\end{array}$ & $\begin{array}{l}\text { Uma das características principais de inteligência no } \\
\text { setor público está na necessidade de aproveitar efetiva- } \\
\text { mente as habilidades e conhecimentos que, muitas vezes, } \\
\text { estão latentes na multidão, e buscar a colaboração e par- } \\
\text { ticipação das pessoas na busca pela eficiência e eficácia } \\
\text { da atividade pública. Pessoas são as receptoras de dados } \\
\text { e informações advindas do monitoramento do ambiente. }\end{array}$ \\
\hline $\begin{array}{l}\text { Uso de Tec- } \\
\text { nologia da } \\
\text { Informação } \\
\text { e Comuni- } \\
\text { cação }\end{array}$ & $\begin{array}{l}\text { De Paula e Rover (2012); } \\
\text { Gil-Garcia, Pardo e Aldama- } \\
\text {-Alda (2013); } \\
\text { Gil-Garcia, Zhang e Puron- } \\
\text {-Cid (2016); Johnston e Han- } \\
\text { sen (2011); Scholl e Scholl } \\
\text { (2014) }\end{array}$ & $\begin{array}{l}\text { O uso da tecnologia da informação e comunicação } \\
\text { para a coleta de dados do ambiente externo. Auxílio no } \\
\text { compartilhamento de informações entre as partes inte- } \\
\text { ressadas e tecnologia como meio facilitador na capta- } \\
\text { ção de dados e informações acionáveis e onipresentes } \\
\text { no ambiente. }\end{array}$ \\
\hline $\begin{array}{l}\text { Objetivos e } \\
\text { relevância } \\
\text { da Inteli- } \\
\text { gência para } \\
\text { a gestão } \\
\text { pública }\end{array}$ & $\begin{array}{l}\text { Gil-Garcia, Pardo e Aldama- } \\
\text {-Alda (2013); } \\
\text { Gil-Garcia, Zhang e Puron- } \\
\text {-Cid (2016); Johnston e Han- } \\
\text { sen (2011); Scholle Scholl } \\
\text { (2014) }\end{array}$ & $\begin{array}{l}\text { Propiciar a tomada de decisão do gestor público ba- } \\
\text { seada em dados e informações pertinentes acarretando } \\
\text { vantagens para a Administração pública: melhorar a } \\
\text { prestação de serviços públicos, superar desafios com- } \\
\text { plexos, tornar a gestão pública mais ágil, melhorar a } \\
\text { eficácia das políticas e programas públicos. }\end{array}$ \\
\hline
\end{tabular}
Fonte: os autores.

A partir do Quadro 1, é possível depreender que os aspectos relacionados e identificados a partir da revisão teórica em relação à inteligência na gestão pública remetem a questões que envolvem diretamente a cultura organizacional, como a maneira como a gestão incentiva a participação das pessoas nos processos, a influência da tecnologia no desenvolvimento da atividade de inteligência e a forma com que os dados e informação são disseminados e compartilhados nas organizações públicas. Além de questões tecnológicas e processuais relacionadas à inteligência na gestão pública, a partir da revisão realizada foi possível adicionalmente depreender uma relação aproximada e determinante de elementos ligados às pessoas, como habilidades, conhecimentos, experiências, compartilhamento, uso, motivação e participação das mesmas na busca pela eficiência e eficácia da atividade pública. Como parte do sis- 
tema, pessoas são as receptoras de dados e informações advindas do monitoramento do ambiente, bem como as mediadoras das atividades realizadas. Evidencia-se, assim, a relevância de compreender na prática administrativa do setor público tal influência para a concretização de processos de inteligência, especialmente quanto ao apoio da alta gestão no desenvolvimento de processos de inteligência, o papel das pessoas nos referidos processos e a influência da tecnologia na concretização dos mesmos.

\section{PROCEDIMENTOS METOdOLÓGICOS}

Considerando o objetivo neste artigo de identificar elementos relacionados à influência da cultura organizacional no desenvolvimento de processos de inteligência na gestão pública, optou-se pela abordagem qualitativa e exploratória uma vez que "um dos principais benefícios da pesquisa qualitativa é que ela permite que um pesquisador veja e compreenda o contexto no qual as decisões e ações ocorrem.” (MYERS, 2013, p. 5). Além disso, segundo o referido autor, pesquisadores qualitativos argumentam que, se você quiser entender as motivações das pessoas, suas razões, suas ações e o contexto de suas crenças e ações de forma aprofundada, a pesquisa qualitativa é a escolha adequada. É exploratória em razão de que "um dos principais benefícios da pesquisa qualitativa é que ela permite que um pesquisador veja e compreenda o contexto no qual as decisões e ações ocorrem.” (MYERS, 2013, p. 5).

Nesta pesquisa, após revisão da literatura sobre os dois principais temas de interesse - cultura organizacional e inteligência na gestão pública -, foi consolidado um conjunto de elementos (Quadro 1) que embasaram um roteiro para a realização de entrevistas com gestores públicos. Segundo Triviños (1987), a entrevista semiestruturada tem como característica questionamentos básicos apoiados em teorias que se relacionam com o tema da pesquisa. Para o autor, a entrevista semiestruturada favorece não somente a descrição dos fenômenos sociais, mas também sua explicação e a compreensão de sua totalidade, bem como a manutenção da presença consciente e atuante do pesquisador no processo de coleta de informações.

Com base no roteiro, foram realizadas 17 entrevistas com gestores públicos de diferentes órgãos públicos, no período de janeiro até maio de 2017. Os entrevistados foram selecionados por conveniência, desde que atendessem ao requisito de ser servidor de carreira no exercício de atividades de gestão. Importante ressaltar que nenhum dos entrevistados pertence ao alto escalão do Governo, constituindo-se de gestores de diversas áreas dentro de diferentes órgãos. 
Como no contexto da administração pública são inúmeras as áreas de atuação, desde atividades vinculas à gestão da própria máquina pública como aquelas vinculadas à prestação de serviço público para a sociedade, optou-se por não distinguir gestores públicos de uma área específica. Para estabelecimento do contato para realização das entrevistas, foi realizado contato via e-mail ou telefônico com os gestores públicos identificados, indicando os objetivos da pesquisa, o assunto e a duração previstos para as entrevistas. Ao realizar as entrevistas, foram observados e registrados os aspectos (textuais e também percepções) que se destacaram no decorrer da conversa; por fim, foi realizada a transcrição de cada entrevista, na sua totalidade.

Para os procedimentos de análise de dados, optou-se pelo uso da técnica de análise de conteúdo. Conforme Bardin (2011, p. 48), é possível definir o termo análise de conteúdo como

\footnotetext{
um conjunto de técnicas de análise das comunicações visando obter por procedimentos sistemáticos e objetivos de descrição do conteúdo das mensagens indicadores (quantitativos ou não) que permitam a inferência de conhecimentos relativos às condições de produção/recepção (variáveis inferidas) dessas mensagens.
}

A análise das entrevistas partiu da categorização e codificação dos dados em três categorias principais: o apoio da alta gestão no desenvolvimento de processos de inteligência, o papel das pessoas nos referidos processos e a influência da tecnologia na concretização dos mesmos. As três categorias estão vinculadas a questões de cultura organizacional, uma vez que, segundo Johann (2004, p. 5) “é preciso estimular a adaptabilidade da cultura em relação às mudanças do macroambiente”, uma vez que há necessidade de uma liderança forte, porém não repressora das iniciativas nos níveis organizacionais mais baixos. Os resultados da pesquisa, desenvolvidos a partir das análises evocadas, estão expostos a seguir.

\section{APRESENTAÇÃO E DISCUSSÃO DOS RESULTADOS}

Diversas questões relacionadas à influência da cultura organizacional no processo de inteligência na gestão pública puderam ser percebidas com a realização das entrevistas com os gestores públicos, como a influência do apoio da alta gestão, a participação dos servidores no processo, o incentivo do uso de TICs. Quanto à caracterização dos entrevistados, a maior parte deles exerce funções de coordenação, gerência e direção. Possuem mais de 10 anos no serviço público, com formação em 
nível de pós-graduação. Entre as áreas de atuação, destacam-se aquelas vinculadas ao planejamento e gestão, qualidade do gasto público, projetos e sistemas e fiscalização e cobrança. Para apresentação dos resultados, a análise está estruturada conforme a relação dos elementos consolidados no Quadro 1, a saber: apoio da alta gestão, capital humano, uso da Tecnologia da Informação e Comunicação e objetivos e relevância dos processos de inteligência na gestão pública.

\subsection{APOIO DA ALTA GESTÃO}

A partir da análise das entrevistas, foi possível perceber que a cultura relacionada à necessidade do uso de dados e informações advindos do monitoramento do ambiente externo, inerentes à atividade de inteligência, começou a ser disseminada com o decorrer dos anos, corroborando a questão de que um processo de mudança nos padrões culturais da organização não admite rupturas bruscas, mas transformações adaptativas operadas em vetores essenciais da estrutura (BERGUE, 2011).

Ressalta-se que devemos considerar o apoio da gestão como aquele vinculado a cada departamento e setor de análise, e não ao alto escalão de governo. De fato, até o presente momento, desconhece-se a existência de um movimento de governo (como um todo) no sentido de captar, analisar e utilizar ainda mais dados e informações, por mais que se reconheça a importância dos mesmos para uma gestão pública qualificada.

Conforme a visão da totalidade dos entrevistados, por muito tempo a administração pública possuiu uma visão endêmica, atendo-se a sua própria produção de dados e informações em vez de explorar o que é produzido e está disponível no ambiente. Entretanto, alegam que a partir da última década, esse posicionamento começou a evoluir para uma preocupação com os dados e informações do ambiente externo para a tomada de decisão enquanto gestores.

Outro ponto que merece destaque é em relação à cultura organizacional forte (ROBBINS; JUDGE, 2012) da administração pública, uma vez que a maioria dos servidores possui as mesmas opiniões sobre o modo de operar da organização, vinculado, por vezes, às questões de traços de paternalismos (BERGUE, 2010), acarretando barreira às mudanças de atitudes e comportamentos em busca de uma melhor gestão pública. Para a totalidade dos gestores entrevistados, a preocupação e interesse com dados e informações do ambiente externo ficam muito individualizadas, não havendo a permeabilidade pela organização como um todo que esteja funcionando tão bem que possa ser um padrão organizacional. 
Conforme exposto por um dos entrevistados, a adaptação de processos de inteligência passa pela cultura, inclusive dos servidores, e que o nível de compartilhamento das informações e engajamento está crescendo de maneira contínua, principalmente pela valorização da questão por parte da equipe diretiva, a qual está apoiando e compreende a importância do tema como gerador de eficiência para a administração pública.

Quanto ao apoio da alta gestão, tratado como um dos pontos centrais da presente análise, 15 dos 17 gestores entrevistados entendem que é a partir do estímulo do gestor de cada área que é possível a concretização de processos de inteligência. Entretanto, ressaltam que a alta gestão, geralmente, é efêmera, não durando mais do que quatro anos. Essa questão do turnover dificulta a estruturação de processos, ficando a preocupação na gestão interna de cada setor ou órgão. Contudo, os entrevistados alegam que existe o incentivo no sentido de dar a liberdade de melhorar o processo e buscar que ele possa permear a administração pública como um todo.

Segundo cinco gestores entrevistados, a passo de estar havendo uma mudança geracional de servidores junto aos órgãos públicos, notou-se uma maior preocupação por parte dos gestores em relação ao desenvolvimento da atividade de inteligência, bem como em relação a como uma maior utilização de dados e informações poderia ser benéfica para uma melhor tomada de decisão e maior eficiência e eficácia nas políticas e programas públicos. A preocupação se estende para a transformação desses dados e informações em conhecimentos úteis, disponíveis e disseminados na organização, mas alegam que ainda há uma dificuldade imensa na disseminação dos dados, informações e conhecimento entre os diversos setores de um mesmo órgão público. Para Desouza (2005), o conhecimento é importante por se tratar da coleta do significado a partir dos dados e informações advindas do ambiente, que precisa ser disseminado entre os diversos setores.

Evidencia-se nas entrevistas a importância exposta na teoria em relação ao apoio da alta gestão para o desenvolvimento de processos de inteligência (GIL-GARCIA; PARDO; ALDAMA-NALDA, 2013; GIL-GARCIA; ZHANG; PURON-CID, 2016; JOHNSTON; HANSEN, 2011; SCHOLL; SCHOLL, 2014). Percebe-se, adicionalmente, a existência de uma relação da prática administrativa de desenvolvimento de processos e atividades de inteligência na gestão pública.

\subsection{O PAPEL DAS PESSOAS}

Quanto à participação dos servidores e o papel destes na disseminação de uma cultura voltada para o monitoramento do ambiente externo, a partir da totalidade 
das entrevistas realizadas, evidenciou-se que essa preocupação fica atrelada aos servidores que exercem cargos de chefia e gestão, ou àqueles que desenvolvam alguma atividade específica que demanda dados e informações externas ao ambiente público. Segundo sete gestores entrevistados, grande parte dos servidores fica no operacional, e é necessário o estímulo da chefia a cada pessoa, pois ainda não é algo consolidado na administração pública. Não é algo que, conforme Russo (2010), já faça parte da cultura e possa determinar o comportamento de modo que poderia ser considerado como internalizado, se tornando inconsciente a ponto de ser realizado de forma natural.

Contudo, todos os gestores reconhecem a importância da participação de cada servidor no processo de internalização de uma cultura voltada para o monitoramento do ambiente e para a utilização de dados e informações externas de importância para o desenvolvimento da atividade pública, uma vez que, para eles, o engajamento dos servidores nos processos de inteligência é fundamental para que o compartilhamento seja, de fato, efetivo. Para os entrevistados, servidores engajados tendem a tornar o processo uma prática rotineira e positiva dentro da organização, gerando crescimento para todos, além disso, alegam que nos setores que essa cultura está mais disseminada, já existem resultados positivos no desenvolvimento da atividade pública a partir de processos de inteligência.

Além disso, a partir das entrevistas, foi possível depreender que o papel das pessoas é indispensável e faz a diferença para a adoção e disseminação de novos processos na gestão pública. Isso corrobora o exposto na teoria de que governo inteligente está vinculado, entre outros aspectos, à partilha de informação aberta e ao uso das informações, à participação das partes interessadas e à colaboração, objetivando a melhoria das operações e serviços governamentais (SCHOLL; SCHOLL, 2014). Nessa linha, cinco entrevistados alegam que não existe mais espaço para um servidor público que ficar à margem desse processo; ele deve ser identificado e integrado ao novo contexto de gestão.

Outro ponto que é destacado pela totalidade dos gestores entrevistados em relação ao papel das pessoas no desenvolvimento de processos de inteligência em governo está vinculado ao desafio em mobilizar cada servidor público a participar do processo de discussão e construção. Alega-se que pelas características inerentes à cultura pública, por vezes o servidor fica na zona de conforto, limitado às questões legais, sem buscar insights advindos do ambiente para a qualificação das atividades a serem realizadas e, consequentemente, para uma melhor gestão pública.

A cultura do setor público ainda é exposta pelos gestores como uma barreira em razão da estrutura burocrática que, por vezes, acaba por não incentivar uma 
maior participação das pessoas em relação à disseminação de dados, informações e conhecimento, ficando muito atrelada às questões unicamente legais do processo, não buscando inovação para a gestão pública, qualificação na tomada de decisão e maior eficiência e eficácia no desenvolvimento das atividades públicas.

Por fim, cabe ressaltar que o estímulo à participação de cada pessoa e à disseminação de uma cultura voltada para o monitoramento do ambiente é exposto pelos gestores públicos como um processo em constante evolução e que já está trazendo bons resultados nas iniciativas por parte de alguns setores específicos de trabalho, corroborando o exposto por Johston e Hansen (2011) no que se refere ao aproveitamento efetivo de habilidades e conhecimentos que muitas vezes estão latentes no ambiente.

\subsection{USO DE TECNOLOGIAS DA INFORMAÇÃO E COMUNICAÇÃO}

O uso de tecnologias da informação e comunicação como aspecto disseminado na cultura da gestão pública e de importância para o processo de inteligência ficou evidente no decorrer da análise das entrevistas. Esse posicionamento corrobora o exposto por De Paula e Rover (2012), Johnston e Hansen (2011) e Schooll e Scholl (2014), no sentido do uso de TICs para coleta de dados do ambiente externo, bem como o auxílio no compartilhamento de informações entre setores e pessoas.

O aumento do uso de TICs por parte do setor público é exposto como algo disruptivo por parte dos gestores entrevistados, uma vez que tem possibilitado o processamento de grandes volumes de dados e informações por meio de sistemas informatizados, passando de um governo prescritivo para um processo mais preditivo em atividades de fiscalização, controle do gasto público e compras públicas, uma vez que, a partir dos referidos sistemas, é possível um cruzamento dos inúmeros dados e informações que chegam até o governo. Esse posicionamento vai ao encontro do exposto na teoria sobre a importância do uso de TICs no processo de inteligência para uma maior agilidade e eficiência em governo (GIL-GARCIA; ZHANG; PURON-CID, 2016; JOHSTON; HANSEN, 2011).

Segundo os entrevistados, a cultura de utilização de TICs é inerente tanto para as rotinas de trabalho quanto para a captação e monitoramento de dados e informações do ambiente externo que possam ser processados, mas ressaltam que a alta capacidade de processamento advinda do uso dos sistemas não é suficiente para caracterizar o processo de inteligência na gestão pública em razão de que, pelas regras de processamento, a interpretação desses cruzamentos é inerente à atividade do servidor público, e a tecnologia é apenas um suporte que facilita o trabalho das pessoas. 
Ainda, os gestores entrevistados alegam que o uso da tecnologia em governo é indispensável para trabalhar com o universo e complexidade de dados e informações que chegam até à administração pública, mas reconhecem que o processo de inteligência deveria existir mesmo sem a utilização da mesma. A tecnologia deve ser vista como uma grande ferramenta, mas não como responsável pelo desenvolvimento de processos de inteligência na gestão pública, conforme exposto por um dos entrevistados:

temos recursos de tecnologia e eles são fundamentais para o nosso dia a dia, muito do que a gente está fazendo hoje se apoia em tecnologia de ponta, mas às vezes a gente cai nesse engano de que a tecnologia por si só é a solução, ela é o caminho; a solução está na gestão, na capacidade das pessoas perceberem o caminho a ser tomado. (informação verbal).

Diante do exposto, evidencia-se que para os entrevistados as questões relacionadas à cultura organizacional são impulsionadoras dos processos de inteligência na gestão pública. De fato, verifica-se que a partir do fortalecimento de um contexto estimulado para o monitoramento do ambiente externo, com pessoas engajadas na captura de dados e informações externas ao Governo, seja possível concretizar esses processos na administração pública.

Para os gestores, a evolução da preocupação por parte da gestão pública em relação à coleta, disseminação e uso de dados e informações advindos do ambiente externo para uma melhor tomada de decisão e maior eficiência e eficácia no desenvolvimento da atividade pública, tem se demonstrado fortemente nos últimos anos. Ressaltam, ainda, que tal evolução está atrelada às mudanças que estão ocorrendo na cultura organizacional do setor público a partir de uma maior acessibilidade, interesse e controle da sociedade em relação à eficiência na utilização dos recursos públicos.

Em relação à quarta perspectiva apresentada no Quadro 1, relacionada aos objetivos e relevância da inteligência, a mesma referencia a importância do referido processo para a gestão pública. A seguir, apresentam-se as considerações finais do presente estudo, evidenciando-se as limitações e oportunidades de futuras pesquisas sobre a temática de estudo.

\section{CONSIDERAÇÕES FINAIS}

No presente estudo buscou-se identificar pontos importantes relacionados à influência da cultura organizacional na concretização de processos de inteligência na 
gestão pública. Os principais achados remetem que a cultura organizacional impulsiona a administração pública a monitorar o ambiente externo na busca de dados de informações importantes e de relevância para a tomada de decisão dos gestores públicos, bem como para uma melhor eficiência e eficácia no desenvolvimento da atividade pública.

Entretanto, a totalidade dos gestores públicos entrevistados apontam que o processo de inteligência ainda não se encontra disseminado, nem pode, ainda, ser considerado parte integrante da cultura organizacional do setor público. Eles reconhecem, contudo, que existe uma forte evolução da preocupação de governo, principalmente na última década, atrelada com a intensificação do uso de TICs na gestão pública, com a captação, produção, utilização e disseminação de dados e informações do ambiente externo.

Outro ponto que merece destaque é o papel fundamental do apoio da alta gestão para disseminação da cultura voltada para implantação de processos de inteligência, o engajamento dos servidores públicos em tais processos, bem como a tecnologia da informação aparecendo fortemente como um mecanismo facilitador de captação e disseminação de dados e informações nas organizações públicas.

Pode-se concluir que ainda existe a necessidade de mexer com as bases da cultura organizacional na gestão pública que permeia toda a organização, buscando um maior incentivo à concretização de processos de inteligência. Apenas algumas iniciativas pontuais estão conseguindo desenvolver esses processos, o que pode ser compreendido, como exposto por Robbins (2005, p. 377), como subculturas que refletem experiências de alguns membros ou setores da organização, as quais não podem ser desconsideradas e nem descartadas pelo gestores. Tais subculturas, voltadas para os processos de inteligência, podem potencialmente ser norteadoras de uma disseminação maior, tornando-se uma cultura dominante dentro do setor público.

Compreende-se a importância da influência da cultura na organização para a estruturação, disseminação e perpetuação dos processos de inteligência na gestão pública. Ainda, considera-se que o enraizamento da visão voltada para a utilização de dados e informações advindos do ambiente por meio de processos de inteligência na cultura organizacional da gestão pública, possibilita uma maior qualidade na tomada de decisão dos gestores públicos e no desenvolvimento efetivo da atividade pública, independentemente de eventuais trocas de governo.

Espera-se, com este estudo, ter contribuído conceitualmente com questões vinculadas à cultura organizacional e a sua influência no desenvolvimento de processos de inteligência na gestão pública. Para a gestão pública, acredita-se no sentido de 
produzir ideias e estímulos em relação à importância de existir uma cultura voltada para o monitoramento do ambiente externo e da utilização de dados e informações advindos dele para a produção de conhecimento na administração pública como meio de melhoria da gestão.

A dificuldade em se aproximar de áreas de inteligência vinculadas à gestão pública, bem como a negativa para realização de entrevistas com gestores que trabalham nas referidas áreas caracteriza-se como limitação do presente estudo, além disso, a participação de órgãos de um único estado do Brasil no estudo não possibilitou a realização de comparativos entre culturas organizacionais distintas.

Sugere-se, para futuras pesquisas, uma análise sobre os servidores que não exercem cargo de gestão, buscando compreender quais fatores da cultura organizacional impulsionam ou barram o desenvolvimento de processos de inteligência na gestão pública. Ainda, realizar estudos em áreas específicas de inteligência em governo buscando insights importantes das referidas subculturas para o sucesso do processo.

\section{REFERÊNCIAS}

AGUILAR, F. J. Scanning the Business Environment. New York: Macmillan, 1967.

AGUNE, R.; CARLOS, J. Governo eletrônico e novos processos de trabalho. In: LEVY, E.; DRAGO, P. Gestão pública no Brasil contemporâneo. São Paulo: Fundap, 2005. p. 302-315.

ANDRIOTTI, F. K.; FREITAS, H. M. R. de; JANISSEK-MUNIZ, R. Informação informal e a monitoração do ambiente organizacional: reflexões e sugestões para a área de TI. In: ENANPAD, 32., 2008, Rio de Janeiro. Anais... Rio de Janeiro: ANPAD, 2008.

AUSTER, E.; CHOO, C. W. How senior managers acquire and use information in environmental scanning. Information Processing \& Management, v. 30, i. 5, p. 607-618, 1994.

BARDIN, L. Análise de conteúdo. Lisboa: Edições 70, 2011. 
BATISTA, F. F. Modelo de gestão do conhecimento para a administração pública brasileira: como implementar a gestão do conhecimento para produzir resultados em benefício do cidadão. Brasília, DF: Ipea, 2012. Disponível em: <http://repositorio.ipea.gov.br/bitstream/11058/754/1/Modelo\%20de\%20Gest\%c3\%a3o\%20do\%20 Conhecimento\%20para\%20a\%20Administra\%c3\%a7\%c3\%а3о\%20P\%c3\%bablica\%20Brasileira.\%20Livro.pdf>. Acesso em: 06 jun. 2017.

BERGUE, S. T. Comportamento organizacional. Florianópolis: CAPES, UAB, 2010.

BERGUE, S. T. Modelos de Gestão em Organizações Públicas: teorias e tecnologias para análise e transformação organizacional. Caxias do Sul: Educs, 2011.

BRÂNZAŞ, B. V.; RADU, I. Transformation of Public Management Process Due to Competitive Intelligence Implementation. Procedia Economics and Finance, v. 32, p. 694-701, 2015.

CAPOBIANGO, R. P. Reformas administrativas no Brasil: uma abordagem teórica e crítica. REGE - Revista de Gestão, v. 20, n. 1, p. 61-78, 2013.

CEPIK, M. Inteligência, política e poder no Estado contemporâneo. Revista de Sociologia e Política, n. 9, p. 193-196, 1997.

CEPIK, M. Regime político e sistema de inteligência no Brasil: legitimidade e efetividade como desafios institucionais. Dados - Revista de Ciências Sociais, v. 48, n. $1,2005$.

CHOO, C. W. Information management for the intelligent organization: the art of scanning the environment. Medford: Information Today, Inc., 2002.

CHOO, C. W. The Art of Scanning the Environment. Bulletin of the American Society for Information Science and Technology, 1999. p. 21-24.

COOPER, C. L.; CARTWRIGHT, S.; EARLEY, P. C. Organizational culture and climate. Chichester: John Wiley \& Sons Ltda, 2001.

DAVENPORT, T. H. Ecologia da informação: por que só a tecnologia não basta na era da informação. Tradução Bernadette S. Abrão. São Paulo: Futura, 1998. v. 4. 
DE PAULA, A. P. P. Administração pública brasileira entre o gerencialismo e a gestão social. RAE - Revista de administração de empresas, v. 45, n. 1, p. 36-49, 2005.

DE PAULA, G.; ROVER, A. J. O governo eletrônico e a atividade de inteligência. Revista Democracia Digital e Governo Eletrônico, v. 1, n. 6, 2012.

DESOUZA, K. C. Restructuring government intelligence programs: a few good suggestions. Government Information Quarterly, v. 22, n. 3, p. 342-353, 2005.

FERREIRA, M. C. et al. Desenvolvimento de um instrumento brasileiro para avaliação da cultura organizacional. Estudos de Psicologia, v. 7, n. 2, p. 271-280, 2002.

FREITAS, H.; JANISSEK-MUNIZ, R. Uma proposta de plataforma para Inteligência Estratégica. In: CONGRESSO IBERO-AMERICANO DE GESTÃO DO CONHECIMENTO E INTELIGÊNCIA COMPETITIVA, 1., 2006, Curitiba. Anais... Curitiba: ABRAIC, 2006.

GIL-GARCIA, J. R.; HELBIG, N.; OJO, A. Being smart: Emerging technologies and innovation in the public sector. Government Information Quarterly, v. 31, p. 11-18, 2014.

GIL-GARCIA, J. R.; PARDO, T. A.; ALDAMA-NALDA, A. Smart cities and smart governments: using information technologies to address urban challenges. In: ACM INTERNATIONAL CONFERENCE PROCEEDING SERIES, 14., 2013, Canadá. Proceedings... Canadá: ACM, 2013.

GIL-GARCIA, J. R.; ZHANG, J.; PURON-CID, G. Conceptualizing smartness in government: An integrative and multi-dimensional view. Government Information Quarterly, v. 33, i. 3, p. 524-534, 2016.

HAKANSSON, C.; NELKE, M. Competitive Intelligence for Information Professionals. Whaltham: Chandos Publishing, 2015.

HERMAN, M. Intelligence power in peace and war. Cambridge: Cambridge University Press, 1996.

HOFSTEDE, G. Cultures and organizations: software of the mind. London: McGrawHill UK, 1991. 
JANISSEK-MUNIZ, R. Fatores Críticos em Projetos de Inteligência Estratégica Antecipativa e Coletiva. Revista Inteligência Competitiva, v. 6, n. 2, p. 147-180, 2016.

JANISSEK-MUNIZ, R.; BLANCK, M. R. de M. Weak signals management, entrepreneurship and uncertainty: a relational theoretical essay under the perspective of intelligence. In: INTERNATIONAL CONFERENCE ON INFORMATION SYSTEMS AND TECHNOLOGY MANAGEMENT - CONTECSI, 11., 2014, São Paulo. Anais... São Paulo: CONTECSI, 2014.

JIMENEZ, C. E.; SOLANAS, A.; FALCONE, F. E-government interoperability: Linking open and smart government. Computer, v. 47, i. 10, p. 22-24, 2014.

JOHANN, S. L. Gestão da cultura corporativa: como as organizações de alto desempenho gerenciam sua cultura organizacional. São Paulo: Saraiva, 2004.

JOHNSTON, E. W.; HANSEN, D. L. Design lessons for smart governance infrastructures. In: BALUTIS, A. P.; INK, D. Transforming American governance: Rebooting the public square. National Academy of Public Administration, 2011. p. 197-212.

KLERING, L.; PORSSE, M. de C. S.; GUADAGNIN, L. A. Novos caminhos da administração pública brasileira. Análise, v. 21, n. 1, jan./jun. 2010.

LESCA, H. Veille stratégique: la méthode L.E.SCAnning ${ }^{\circledR}$. 1. ed. Grenoble France: Editions EMS, 2003.

LINDERS, D.; LIAO, C. Z. P.; WANG, C. M. Proactive e-Governance: Flipping the service delivery model from pull to push in Taiwan. Government Information Quarterly, 2015. http://doi.org/10.1016/j.giq.2015.08.004

LIU, X.; ZHENG, L. Cross-departmental collaboration in one-stop service center for smart governance in China: Factors, strategies and effectiveness. Government Information Quarterly, 2015. http://dx.doi.org/10.1016/j.giq.2015.12.001.

MELATI, C.; JANISSEK-MUNIZ, R. Knowledge Management and Intelligence: an analysis of conceptual complementarity through the Public Management Perspective. In: CONGRESSO INTERNACIONAL DE GESTÃO DE TECNOLOGIA E SISTEMAS DE INFORMAÇÃO - CONTECSI, 2017, São Paulo. Anais... São Paulo: CONTECSI, 2017. 
MYERS, M. D. Qualitative research in business and management. London: Sage, 2013.

OSBORNE, D.; PLASTRIK, P. Banishing Bureaucracy: The Five Strategies for Reinventing Government. New York: Plume; Addison-Wesley Publishing Company, Inc., 1997.

PRESCOTT J.; MILLER, S. Inteligência competitiva na prática. Rio de Janeiro: Campus, 2002.

REZENDE, D. Projeto de Organizational Business Intelligence (OBI): modelo organizacional de três organizações paranaenses. In: INTERNATIONAL CONFERENCE ON INFORMATION SYSTEMS AND TECHNOLOGY MANAGEMENT - CONTECSI, 2012, São Paulo. Anais... São Paulo: CONTECSI, 2012.

RIBEIRO, L. M. P.; PEREIRA, J. R.; BENEDICTO, G. C. As reformas da administração pública brasileira: uma contextualização do seu cenário, dos entraves e das novas perspectivas. In: ENCONTRO DA ASSOCIAÇÃO NACIONAL DE PÓS-GRADUAÇÃO EM ADMINISTRAÇÃO, 37., 2013, Rio de Janeiro. Anais... Rio de Janeiro: ANPAD, 2013.

ROBBINS, S. P. Comportamento Organizacional. Tradução Reynaldo Marcondes. 11. ed. São Paulo: Pearson Prentice Hall, 2005.

ROBBINS, S. P.; JUDGE, T. A. Organizational Behavior. 15. ed. New Jersey: Prentice Hall, 2012.

RODRIGUES, C.; BLATTMANN, U. Gestão da informação e a importância do uso de fontes de informação para geração de conhecimento. Perspectivas em Ciência da Informação, v. 19, n. 3, p. 4-29, 2014.

RUSSO, G. M. Diagnóstico da cultura organizacional. Rio de Janeiro: Elsevier, 2010.

SCHEIN, E. Cultura Organizacional e liderança. São Paulo: Atlas, 2010

SCHOLL, H. J.; SCHOLL, Margit C. Smart governance: a roadmap for research and practice. In: iCONFERENCE 2014. Proceedings..., 2014.

SECCHI, L. Modelos organizacionais e reformas da administração pública. RAP Revista de Administração pública, v. 43, n. 2, p. 347-369, 2009. 
TRIVIÑOS, A. Introdução à pesquisa em ciências sociais: a pesquisa qualitativa em educação. São Paulo: Atlas, 1987.

VERONESE, J. E. Lei de Acesso à Informação e os reflexos sobre a produção de Inteligência na Polícia Federal. Revista Brasileira de Inteligência, Brasília, DF, n. 8, 2013.

VIDIGAL, F.; ZIVIANI, F. Inteligência Competitiva: Histórico, evolução e metodologias organizacionais sob a ótica da ciência da informação. In: ENCONTRO NACIONAL DE PESQUISA EM CIÊNCIA DA INFORMAÇÃO, 16., 2015, João Pessoa. Anais... João Pessoa, 2015.

WARRICK, D. D.; MILLIMAN, J. F.; FERGUSON, J. M. Building high performance cultures. Organizational Dynamics, v. 1, i. 45, p. 64-70, 2016.

\section{Como citar este artigo:}

\section{ABNT}

MELATI, Cláudia; JANISSEK-MUNIZ, R. A cultura organizacional como impulsionadora dos processos de inteligência na gestão pública. RACE, Revista de Administração, Contabilidade e Economia, Joaçaba: Ed. Unoesc, p. 131-156, 2017. Edição Especial. Disponível em: <http://editora.unoesc.edu.br/index.php/race>. Acesso em: dia/mês/ano.

APA

Melati, C., \& Janissek-Muniz, R. (2017). A cultura organizacional como impulsionadora dos processos de inteligência na gestão pública [E. especial]. RACE, Revista de Administração, Contabilidade e Economia, 131-156. Recuperado em dia/mês/ano, de http://editora.unoesc.edu.br/index.php/race 